\title{
SOME COMPACTNESS THEOREMS OF FAMILIES OF PROPER HOLOMORPHIC CORRESPONDENCES
}

\author{
NABIL OURIMI* \\ Abstract \\ In this paper we prove some compactness theorems of families \\ of proper holomorphic correspondences. In particular we extend \\ the well known Wong-Rosay's theorem to proper holomorphic cor- \\ respondences. This work generalizes some recent results proved \\ in $[\mathbf{1 7}]$.
}

\section{Introduction and results}

In [29], B. Wong gave a characterization of the unit ball in $\mathbb{C}^{n}$ by its automorphism group, namely, if $D$ is a smooth strongly pseudoconvex bounded domain in $\mathbb{C}^{n}$ with noncompact automorphism group, then $D$ is biholomorphic to the unit ball. Later, J.-P. Rosay [21] proved that the same conclusion holds under considerably weaker hypotheses on the boundary of the domain. S. Pinchuk [19] gave a local version of this theorem with an elementary proof by using the scaling technique: the unit ball is a model for the class of $C^{2}$ strongly pseudoconvex domains at an accumulation point. E. B. Lin and B. Wong [14] observed that this result (termed "the Wong-Rosay theorem") is interesting only when the domain $D$ is an Eilenberg-Maclane space (i.e. $\pi_{k}(D)=0$ for all $k \geq 1$ ); since a smooth bounded domain $D$ in $\mathbb{C}^{n}$ with noncompact automorphism group and nontrivial $\pi_{k}(D)$ for some $k \geq 1$ admits a complex analytic variety in the boundary. In particular, they proved that the set of proper holomorphic mappings between bounded strongly pseudoconvex domains in $\mathbb{C}^{n}$ is noncompact if both of the domains are biholomorphic to the unit ball. In [17], the author showed a local version of this result: if $D$ is a bounded domain in $\mathbb{C}^{n}$ and there exist a point $p \in D$ and a sequence of proper holomorphic self-mappings $f_{k}: D \rightarrow D$ of uniformly

2000 Mathematics Subject Classification. 32H35.

Key words. Proper holomorphic correspondences, scaling technique.

*The research was supported by the ICTP, Trieste, Italy. 
bounded multiplicity such that $\left\{f_{k}(p)\right\}_{k}$ converges to a strongly pseudoconvex boundary point, then $D$ is biholomorphic to the unit ball in $\mathbb{C}^{n}$. Our aim in this paper is to prove a suitable version of the Wong-Rosay theorem for families of proper holomorphic correspondences.

The notion of holomorphic correspondence is very interesting. It is a generalization to several complex variables of the classical global analytic function of one complex variables. More precisely, let $D$ and $G$ be two domains in $\mathbb{C}^{n}$. A holomorphic correspondence is a closed complex analytic subset $A \subset(D \times G)$ of pure dimension $n$ with $\bar{A} \cap(D \times \partial G)=\emptyset$. We can regard $A$ as the graph of the multivalued mapping $f:=\pi_{2} \circ \pi_{1}^{-1}$, where $\pi_{1}: A \rightarrow D$ and $\pi_{2}: A \rightarrow G$ denote the natural projections. It follows from the definition that the projection $\pi_{1}: A \rightarrow D$ is proper. Then there exists an $n$-1-dimensional analytic subset $V \subset$ graph $f$ and an integer $m$, such that $\pi_{1}$ is an $m$-sheeted covering map from the set $A \backslash \pi_{1}^{-1}\left(\pi_{1}(V)\right)$ onto $D \backslash \pi_{1}(V)$. Hence $f(z)=\left\{f^{1}(z), \ldots, f^{m}(z)\right\}$ for all $z \in D \backslash \pi_{1}(V)$ and the $f^{j}$ 's are distinct holomorphic functions in a neighborhood of $z \in D \backslash \pi_{1}(V)$. The integer $m$ is called the multiplicity of $f$ and $\pi_{1}(V)$ is its branch locus. If both $\pi_{1}$ and $\pi_{2}$ are proper then $A$ is a proper holomorphic correspondence. If $A$ is irreducible as an analytic set, then it is called an irreducible holomorphic correspondence. For the basic topic on holomorphic correspondences, we refer the reader to the work of K. Stein $[\mathbf{2 5}],[\mathbf{2 6}]$ and for its boundary behavior to $[\mathbf{1}],[\mathbf{6}]$ and [27], where the phenomena of continuous and holomorphic extension for correspondences were studied with local boundary assumptions.

We denote by $\operatorname{Cor}(D, G, m)$ the set of all $\mu$-valued holomorphic mappings from $D$ onto $G$ for $\mu=1, \ldots, m$ and $\operatorname{Cor}(D, G, m, l)$ the set of correspondences $f \in \operatorname{Cor}(D, G, m)$ for which $f^{-1} \in \operatorname{Cor}(G, D, l)$.

Our main result can be stated as follows:

Theorem 1. Let $D$ and $G$ be bounded domains in $\mathbb{C}^{n}$. Suppose that there exist a point $p \in D$, a sequence of proper holomorphic irreducible correspondences $\left\{f_{k}\right\}_{k}$ in $\operatorname{Cor}(D, G, m, l)$ and a sequence $\left\{q_{k}\right\}_{k}, q_{k} \in$ $f_{k}(p)$, converging to a strongly pseudoconvex boundary point $q \in \partial G$. Then there exists a proper holomorphic correspondence in $\operatorname{Cor}(D, \mathbb{B}, m, l)$, where $\mathbb{B}$ denotes the unit ball in $\mathbb{C}^{n}$.

In the case $m=1$, we find the result of [17] for proper holomorphic mappings of uniformly bounded multiplicity. If the domain $D$ is a pseudoconvex, simply connected with a $C^{\infty}$ boundary and of finite type (in the sense of J. P. D'Angelo [10]), then the correspondence $f: D \rightarrow \mathbb{B}$ defined by Theorem 1 splits at each point $z \in D$, i.e. $f=\left\langle f_{1}, \ldots, f_{m}\right\rangle$, where the $f_{j}^{\prime} s$ are distinct holomorphic functions in a neighborhood of $z$ 
(see [3]). In view of the simple connectedness we have a global splitting. So each branch of $f$ defines a proper holomorphic mapping from $D$ onto $\mathbb{B}$. In particular any proper holomorphic self-mapping of $D$ is biholomorphic. Moreover the Lie groups $\operatorname{Aut}(D)$ and $\operatorname{Aut}(\mathbb{B})$ have the same dimension (see also [3]). Note that the same conclusions hold if we assume that $G$ is pseudoconvex, simply connected with $C^{\infty}$ boundary and of finite type.

As an application of Theorem 1, we have the following version of the Wong-Rosay theorem for families of proper holomorphic correspondences.

Theorem 2. Let $D$ and $G$ be bounded domains in $\mathbb{C}^{n}$. Assume that $G$ is strongly pseudoconvex, simply connected with a $C^{\infty}$ boundary. Suppose that there exist a point $p \in D$, a sequence of proper holomorphic irreducible correspondences $\left\{f_{k}\right\}_{k}$ in $\operatorname{Cor}(D, G, m, l)$ and a sequence $\left\{q_{k}\right\}_{k}$, $q_{k} \in f_{k}(p)$, converging to a boundary point $q \in \partial G$. Then $G$ is biholomorphic to the unit ball.

Example 1. The following example due to E. Bedford and S. Bell [2], shows that the strong pseudoconvexity in Theorem 2 is necessary. Let $D=\left\{(z, w) \in \mathbb{C}^{2}:|z|^{4}+|w|^{2}<1\right\}$ and let us consider the proper holomorphic mapping

$$
f: \quad \begin{aligned}
D & \rightarrow \mathbb{B} \\
(z, w) & \mapsto\left(z^{2}, w\right) .
\end{aligned}
$$

If $\left\{\varphi_{k}\right\}_{k}$ is a sequence of automorphisms of the unit ball converging to $(1,0) \in \partial \mathbb{B}$ then $\left\{f^{-1} \circ \varphi_{k} \circ f\right\}_{k}$ is a sequence of self-correspondences of $D$ converging to the points $( \pm 1,0) \in \partial D$.

In [12], W. Klingenberg and S. Pinchuk proved that the set of proper holomorphic correspondences of uniformly bounded multiplicity between bounded domains is normal. In strongly pseudoconvex case we get more information on the convergence of such correspondences as follows:

Corollary 1. Let $D$ and $G$ be bounded domains in $\mathbb{C}^{n}$. Assume that at least one of them is strongly pseudoconvex, simply connected with $a C^{\infty}$ boundary and not biholomorphic to the unit ball. Then for any sequence $\left\{f_{k}\right\}_{k}$ of proper holomorphic irreducible correspondences in $\operatorname{Cor}(D, G, m, l)$, we may extract a subsequence converging to a proper holomorphic correspondence in $\operatorname{Cor}(D, G, m, l)$.

This corollary generalizes the result of E. B. Lin and B. Wong [14] mentioned above even for proper holomorphic mappings. 
We can also use Theorem 1 to complete the result of [17], concerning homogeneous complex manifolds.

Corollary 2. Let $M$ be an $n$-dimensional complex homogeneous manifold and $G$ a bounded domain in $\mathbb{C}^{n}$ that possesses strong pseudoconvexity boundary points. Then

(1) There is no proper holomorphic mapping between $M$ and $G$.

(2) If, in addition, $G$ is a strongly pseudoconvex, simply connected domain with a $C^{\infty}$ boundary and there exists a proper holomorphic correspondence between $M$ and $G$, then $G$ is biholomorphic to the unit ball.

\section{Basic facts about convergence of holomorphic correspondences}

In this section we recall some definitions on the notion of convergence of holomorphic correspondence.

Let $z_{o}$ be a point in $D$ and let $\left\{z^{1}, \ldots, z^{m}\right\}$ be a set in $G$. We say that $f(z)=\left\{f\left(z^{1}\right), \ldots, f\left(z^{m}\right)\right\}$ converges to $\left\{z^{1}, \ldots, z^{m}\right\}$ when $z$ tends to $z_{o}$ if after possible renumeration of $f^{j}$, one has $\lim _{z \rightarrow z_{o}} f^{j}(z)=z^{j}$. Equivalently, $f(z)$ tends to $\left\{z^{1}, \ldots, z^{m}\right\}$ in the sense of the Hausdorff convergence of sets. We can also define a distance in $\mathbb{C}^{n m} \times \mathbb{C}^{n m}$ to study the problem of convergence of correspondences (see [5]).

Let $f \in \operatorname{Cor}(D, G, m)$ be an irreducible correspondence, $a \in \mathrm{A} \subset D$ and $b \in f(a)$. We define $f_{\mathrm{A}}^{(a, b)} \in \operatorname{Cor}(\stackrel{\circ}{\mathrm{A}}, G, m)$, where $\stackrel{\circ}{\mathrm{A}}$ refers to the interior of $\mathrm{A}$ as follows:

Consider all irreducible germs of branches of $f$ at $(a, b)$. Analytic continuation of each of these irreducible germs along all possible paths in $\mathrm{A}$ define $f_{\mathrm{A}}^{(a, b)} \in \operatorname{Cor}(\AA, G, m)$. Equivalently, graph $f_{\mathrm{A}}^{(a, b)}$ is the union of those irreducible components of graph $f \cap\{\mathrm{A} \times G\}$, which contains $(a, b)$.

Let $\left\{f_{k}\right\}_{k} \subset \operatorname{Cor}(D, G, m)$. We say that $\left\{f_{k}\right\}_{k}$ is compactly divergent if $\forall K_{1} \subset \subset D, K_{2} \subset \subset G, \exists j_{o}$, such that $\forall j \geq j_{o}$ :

$$
f_{k}\left(K_{1}\right) \cap K_{2}=\emptyset \text {. }
$$

If the $f_{k}$ are irreducible, we say that $f_{k}$ converge to $f \in \operatorname{Cor}(D, G, m)$ if $\exists\left(a, b_{k}\right) \in$ graph $f_{k}$ with $b_{k} \rightarrow b \in G$ and for all $K \subset \subset D$ with $a \in K$ :

$$
f_{k, K}^{(a, b)} \rightarrow f_{K} \text { for some } f_{K} \in \operatorname{Cor}(D, G, m)
$$

and

$$
\cup_{K \subset \subset D} \operatorname{graph} f_{K}=\operatorname{graph} f
$$




\section{Proof of results}

Proof of Theorem 1: Our basic tool is the scaling method, successfully applied in different problems for holomorphic and CR mappings by several authors (see for instance $[\mathbf{1 8}],[\mathbf{4}],[\mathbf{1 1}],[\mathbf{8}],[\mathbf{9}]$ ). It is worth to remark that here we adapt the scaling technique for proper holomorphic correspondences. We believe that this technique will be useful to deal with other problems as well.

We write $z \in \mathbb{C}^{n}$ as $z=\left({ }^{\prime} z, z_{n}\right)$ where ${ }^{\prime} z$ denotes the first $n-1$ coordinates of $z$. Let $V$ be a neighborhood of $q$ in $\mathbb{C}^{n}$ which does not intersect the set of weakly pseudoconvex points of $\partial G$. For all $w \in \partial G \cap V$, we consider the change of variables $h^{w}$ defined by:

$$
\left\{\begin{array}{l}
z_{j}^{*}=\frac{\partial r}{\partial \bar{z}_{n}}(w)\left(z_{j}-w_{j}\right)-\frac{\partial r}{\partial \bar{z}_{j}}(w)\left(z_{n}-w_{n}\right), \quad 1 \leq j \leq n-1 \\
z_{n}^{*}=\sum_{1 \leq j \leq n} \frac{\partial r}{\partial z_{j}}(w)\left(z_{j}-w_{j}\right)
\end{array}\right.
$$

where $r$ is a defining function of $G$. The mapping $h^{w}$ maps $w$ onto 0 and the real normal to $\partial G$ at $w$ onto the line $\left\{{ }^{\prime} z=0, y_{n}=0\right\}$.

Let $w_{k}$ be the point in $\partial G$ closest to $q_{k}$ and $h^{k}=h^{w_{k}}$ be the mapping as above. We denote by $G^{k}=h^{k}(G)$ and $\gamma_{k}=\operatorname{dist}\left(h^{k}\left(q_{k}\right), \partial G^{k}\right)$. We introduce the dilatation of the coordinates as follows: $\beta^{k}\left({ }^{\prime} w, w_{n}\right)=$ $\left(\frac{\prime}{\sqrt[\gamma]{\gamma}_{k}}, \frac{w_{n}}{\gamma_{k}}\right)$. Set $\hat{G}^{k}=\beta^{k} \circ h^{k}(G)$ and consider the holomorphic correspondence $\hat{f}_{k}=\beta^{k} \circ h^{k} \circ f_{k} \in \operatorname{Cor}\left(D, \hat{G}^{k}, m\right)$. Each $\hat{f}_{k}$ is a proper holomorphic correspondence of multiplicity at most $m$ and satisfies $s=$ $\left({ }^{\prime} 0,-1\right) \in \hat{f}_{k}(p)$.

Let $r^{k}$ be a defining function of $G^{k}$. Without loss of generality, we may assume that $q=0$ and in a neighborhood of the origin we have $r(w)=2 R e w_{n}+|w|^{2}+R(w)$ with $R(w)=o\left(|w|^{2}\right)$. By Taylor's formula, we get the estimate $r^{k}(w)=2 R e w_{n}+H^{k}(w)+B^{k}(w)+R^{k}(w)$, where $H^{k}$ is hermitian, $B^{k}$ is bilinear and $R^{k}(w)$ is $o\left(|w|^{2}\right)$ uniformly in a neighborhood of the origin. As $k \rightarrow \infty$, the limit of the matrix $H^{k}$ is the identity and the limit of $B^{k}$ is 0 . Consequently, there exists a neighborhood $U$ of 0 such that for every $k$ and $w \in U$, we have

$$
r^{k}(w) \geq 2 \operatorname{Rew}_{n}+\frac{1}{2}|w|^{2} .
$$

Let $\hat{r}^{k}=\frac{1}{\gamma_{k}} r^{k} \circ \beta^{k^{-1}}$ be a defining function of the domain $\hat{G}^{k}$. It is well known that the sequence $\left\{\hat{r}^{k}(w)\right\}_{k}$ converges uniformly on compact subsets of $\mathbb{C}^{n}$ to $\hat{\varphi}(w)=2 \operatorname{Re}\left(w_{n}\right)+\left.\left.\right|^{\prime} w\right|^{2}$. Let $K$ be an arbitrary compact 
subset of $D$ containing the point $p$. For $z \in K$, let $w^{k} \in \hat{f}_{k, K}^{(p, s)}(z)$. Starting with some $k^{o}=k^{o}(K)$, we have

$$
0>\hat{r}^{k}\left(w^{k}\right)=\frac{1}{\gamma_{k}} r^{k}\left(\sqrt{\gamma}_{k}^{\prime} w^{k}, \gamma_{k} w_{n}^{k}\right) .
$$

To prove the convergence of the correspondence $\left\{\hat{f}_{k}\right\}_{k}$, we shall prove that for large $k$ 's, $\beta^{k^{-1}} \circ \hat{f}_{k, K}^{(p, s)}(K) \subset U$. We need the following important statement on the localization of holomorphic correspondences. It is the crucial point of our scaling construction.

First of all, we recall that a point $a \in \partial D$ is a local plurisubharmonic peak point if there is a neighborhood $V$ of $a$ in $\mathbb{C}^{n}$ and $\psi \in P S H(D \cap$ $V) \cap C(\bar{D} \cap V)$ such that $\psi(a)=1$ and $\psi<1$ on $(\bar{D} \cap U) \backslash\{a\}$. If $V \cap \partial D$ is a strongly pseudoconvex hypersurface of class $C^{2}$, then $a$ is a local plurisubharmonic peak point (see $[\mathbf{2 2}]$ and $[\mathbf{2 4}]$ ).

Proposition 1. Let $D \subset \subset \mathbb{C}^{n}$ and $D^{\prime} \subset \mathbb{C}^{N}, N \geq 1$ be domains. Fix $R>0$ and $z_{o} \in D^{\prime}$. Then for all compact $L \subset D^{\prime}$ containing $z_{o}$, there exists $\delta=\delta(L)>0$ such if $a \in \partial D$ be a local plurisubharmonic peak point of $\partial D, F \in \operatorname{Cor}\left(D^{\prime}, D, m\right)$ and $w \in F\left(z_{o}\right) \cap B(a, \delta)$ then $F_{L}^{\left(z_{o}, w\right)}(L) \subset B(a, R)$.

Proof: This proposition was proved by K. Verma in [27]. For the sake of completeness, we include a brief proof. By contradiction, assume that the proposition is not true. Then there exist a compact $L \subset D^{\prime}$, a sequences $\left\{a_{k}\right\}_{k} \subset \partial D$ and $\left\{F_{k}\right\}_{k} \subset \operatorname{Cor}\left(D^{\prime}, D, m\right)$ with $w_{k} \in F_{k}\left(z_{o}\right) \cap$ $B\left(a_{k}, \delta_{k}\right)$ and $\delta_{k} \rightarrow 0$ such that for all $k, F_{k, L}^{\left(z_{o}, w_{k}\right)}(L) \not \subset B\left(a_{k}, R\right)$. Since the domain $D$ is bounded, we can assume (after taking a subsequence) that $\left\{F_{k}\right\}_{k}$ converges uniformly on compact subsets of $D^{\prime}$ to a holomorphic correspondence $F^{\infty} \in \operatorname{Cor}\left(D^{\prime}, \bar{D}, m\right)$ and $\left\{a_{k}\right\}_{k}$ converges to $a \in$ $\partial D$. Without loss of generality, we may assume that $z_{o}=0^{\prime} \in \mathbb{C}^{N}$ and $a=0^{\prime \prime} \in \mathbb{C}^{n}$. Then we have $\left(0^{\prime}, 0^{\prime \prime}\right) \in\left(D^{\prime} \times \mathbb{C}^{n}\right) \cap$ graph $F^{\infty}$. By using $[\mathbf{7}$, pp. 36 and 46], it easy to see that the graph $F^{\infty}$ is a pure $N$-dimensional analytic set in $D^{\prime} \times \mathbb{C}^{n}$. Let $V^{1}$ be an irreducible component of graph $F^{\infty}$ containing $\left(0^{\prime}, 0^{\prime \prime}\right)$, then again according to $[\mathbf{7}]$ there exist small neighborhoods $U^{\prime} \ni 0^{\prime}, U^{\prime \prime} \ni 0^{\prime \prime}$, so the projection $\pi: V^{1} \cap\left(U^{\prime} \times U^{\prime \prime}\right) \rightarrow U^{\prime}$ is proper.

Let $g_{1}, \ldots, g_{k}$ be the branches of $\pi^{-1}$ which are locally defined and holomorphic on $U^{\prime} \backslash \sigma$, with $\sigma$ an analytic set of dimension at most $N-1$. Since $a=0^{\prime \prime}$ is a local plurisubharmonic peak point, there exist an $\epsilon>0$ and a local plurisubharmonic peak function $\psi \in \operatorname{PSH}\left(B\left(0^{\prime \prime}, \epsilon\right) \cap\right.$ $D) \cap C\left(B\left(0^{\prime \prime}, \epsilon\right) \cap \bar{D}\right)$. Consider $\rho(z)=\max \left(\psi \circ g_{1}(z), \ldots, \psi \circ g_{k}(z)\right) \in$ 
$P S H\left(U^{\prime} \backslash \sigma\right)$. Since $\rho(z)$ is bounded, so it extends as a plurisubharmonic function on $U^{\prime}$. But $\rho\left(0^{\prime}\right)=1$, then by the maximum principle, $\rho(z) \equiv 1$ on $U^{\prime}$. This implies that, one of the branches $g_{i} \equiv 0^{\prime \prime}$ on $U^{\prime}$. Hence $U^{\prime} \times\left\{0^{\prime \prime}\right\} \subset V^{1}$. It follows by irreducibility that $D^{\prime} \times\left\{0^{\prime \prime}\right\}=V^{1}$. Since $V^{1}$ is an arbitrary component containing $\left(0^{\prime}, 0^{\prime \prime}\right)$, it follows that the only one component of graph $F^{\infty}$ containing $\left(0^{\prime}, 0^{\prime \prime}\right)$ is $D^{\prime} \times\left\{0^{\prime \prime}\right\}$. The same argument as above shows that for all $z \in D^{\prime}$, the only component of graph $F^{\infty}$ containing $\left(z, 0^{\prime \prime}\right)$ is $D^{\prime} \times\left\{0^{\prime \prime}\right\}$. Therefore, if $V^{j}$ is some component of graph $F^{\infty}$ distinct from $D^{\prime} \times\left\{0^{\prime \prime}\right\}$, then $V^{j} \cap\left(D^{\prime} \times\left\{0^{\prime \prime}\right\}\right)=\emptyset$. This shows that for all $z \in D^{\prime}, \pi_{2} \circ \pi_{1}{ }^{-1}(z)=\left\{f_{1}^{\infty}(z), \ldots, f_{m-1}^{\infty}(z), 0^{\prime \prime}\right\}$ where $\pi_{1}$ and $\pi_{2}$ are the canonical projections of graph $F^{\infty}$ respectively on $D^{\prime}$ and $\mathbb{C}^{n}$. For all $k$ the branches of $F_{k}$ and $F^{\infty}$ are contained in a bounded domain; hence by the continuity of the root of canonical functions (see [7, pp. 45 and 46]), $m-1$ branches of $F^{\infty}$ are a subset of $D \backslash \bar{B}\left(0^{\prime \prime}, \epsilon_{o}\right)$ while the $m^{\text {th }}$ branch has the constant $0^{\prime \prime}$, for all $z \in L$ and for some constant $\epsilon_{o}>0$. Choose $\hat{\epsilon}_{o}=\min \left(\frac{R}{2}, \frac{\epsilon_{o}}{2}\right)$. Since $\left\{F_{k}\right\}_{k}$ converges uniformly to $F^{\infty}$ on $L$, then for $k \geq k_{o}$ and $z \in L$ all the branches of $F_{k}$ are contained in the disjoint union $B\left(0^{\prime \prime}, \hat{\epsilon}_{o}\right) \cup D \backslash \bar{B}\left(0^{\prime \prime}, \epsilon_{o}-\hat{\epsilon}_{o}\right)$. Since $w_{k} \rightarrow 0$, it follows that for $k \geq k_{o}$ we have $F_{k, L}^{\left(0^{\prime}, w_{k}\right)}(L) \subset B\left(0^{\prime \prime}, \hat{\epsilon}_{o}\right)$. But the sequence $\left\{a_{k}\right\}_{k}$ converges to $0^{\prime \prime}$, then we may assume that for $k \geq k_{o},\left|a_{k}\right|<\frac{R}{2}$. So we have $B\left(0^{\prime \prime}, \hat{\epsilon}_{o}\right) \subset B\left(a_{k}, R\right)$ for all $k \geq k_{o}$. This contradicts the fact that $F_{k, L}^{\left(0^{\prime}, w_{k}\right)}(L) \not \subset B\left(a_{k}, R\right)$.

We continue with the proof of Theorem 1. Let $\alpha>0$ such that $B(0, \alpha) \subset U(U$ is the open set defined in $(*))$ and $K$ a compact in $D$. Let us consider the correspondence $f_{k, K} \in \operatorname{Cor}\left(K^{o}, G, m\right)$. Fix $0<R<\min \left(1, \frac{\alpha}{3}\right)$ and set $\gamma_{k}^{\prime}=\operatorname{dist}\left(q_{k}, \partial D\right)=\left|w_{k}-q_{k}\right|$. Then $q^{k} \in f_{k}(p) \cap B\left(w_{k}, 2 \gamma_{k}^{\prime}\right)$ and for $k$ sufficiently large one has $2 \gamma_{k}^{\prime}<\delta(K)$, where $\delta(K)$ is a constant from Proposition 1. Thus Proposition 1 implies that $f_{k, K}^{\left(p, q_{k}\right)}(K) \subset B\left(w_{k}, R\right)$. Since $h^{k} \rightarrow$ id uniformly on compact subsets of $\mathbb{C}^{n}$ and $w_{k} \rightarrow 0$ as $k \rightarrow \infty$, then for large $k$ 's, we may assume that $B\left(w_{k}, R\right) \subset B(0,2)$ and $\sup _{z \in B(0,2)}\left\|D h^{k}(z)\right\|<3$. Hence $h^{k} \circ f_{k, K}^{\left(p, q_{k}\right)}(L) \subset B(0, \alpha)$. Then for $z \in K$ and $w^{k} \in \hat{f}_{k, K}^{(p, s)}(z)$, we have for large $k$ 's:

$$
\hat{r}^{k}\left(w^{k}\right)>2 \operatorname{Re}\left(w_{n}^{k}\right)+\frac{1}{2}\left|w^{k}\right|^{2} .
$$

Let $\pi_{n}: \mathbb{C}^{n} \rightarrow \mathbb{C}$ be the $n^{\text {th }}$ projection and set $\hat{f}_{k, K}^{n}=\pi_{n} \circ \hat{f}_{k, K}^{(p, s)} \in$ $\operatorname{Cor}(\stackrel{\circ}{K}, \mathbb{H}, m)$, where $\mathbb{H}=\{z \in \mathbb{C}: \operatorname{Re}(z)<0\}$ denotes the half-plane 
in $\mathbb{C}$. Let

$$
\begin{aligned}
T: \mathbb{H} & \rightarrow \Delta \\
z & \mapsto \frac{z+1}{z-1},
\end{aligned}
$$

be the biholomorphic map transforming $\mathbb{H}$ onto the unit disc. Consider now the correspondence $T_{k, K}=T \circ \hat{f}_{k, K}^{n} \in \operatorname{Cor}(\stackrel{\circ}{K}, \Delta, m)$. For all $k$, we have $(s, 0) \in \operatorname{graph} T_{k, K}$. The following statement due to S. Pinchuk [20] is important to prove the convergence of the sequence $\left\{\hat{f}_{k, K}^{n}\right\}_{k}$.

Proposition 2. Let $D$ be a domain in $\mathbb{C}^{n}, n \geq 1$ and $f \in \operatorname{Cor}(D, \Delta, m)$ with $(p, 0) \in \operatorname{graph} f$. Then for all compact $L \subset D$, there exists a constant $r=r(m, L)<1$ not depending on $f$, such that $f_{L}^{(p, 0)}(L) \subset \Delta_{r}$.

According to Proposition 2 and by using Montel's theorem and the diagonal process, we may assume (after taking a subsequence) that $\left\{T_{k, K}\right\}_{k}$ converges to $T_{K} \in \operatorname{Cor}(\stackrel{\circ}{K}, \Delta, m)$. Hence $\hat{f}_{k, K}^{n} \rightarrow \hat{f}_{K}^{n} \in \operatorname{Cor}(\stackrel{\circ}{K}, \mathbb{H}, m)$.

It follows from $(* *)$ that the correspondence $\hat{f}_{k, K}^{(p, s)}$ is uniformly bounded. Then we can extract a subsequence converging to $\hat{f}_{K} \in \operatorname{Cor}\left(\stackrel{\circ}{K}, \mathbb{C}^{n}, m\right)$. By exhausting $D$ with an increasing sequence of compacts containing $p$, we show that $\hat{f}_{k}$ converges to $\hat{f} \in \operatorname{Cor}\left(D, \mathbb{C}^{n}, m\right)$. Passing to the limit in $(* *)$, we conclude that $\varphi(\hat{f})(z) \leq 0$ for all $z \in \Sigma$. Hence $\hat{f} \in$ $\operatorname{Cor}(D, \bar{\Sigma}, m)$.

Now we consider the correspondence $\hat{g}_{k}=\hat{f}_{k}^{-1}$, the inverse of the correspondence $\hat{f}_{k}$. For all $k, \hat{g}_{k} \in \operatorname{Cor}\left(\hat{G}^{k}, D, l\right)$ and satisfies $(s, p) \in$ $\operatorname{graph}\left(\hat{g}_{k}\right)$. Let $L \subset \Sigma$ a compact containing $s$, and consider the correspondence $\hat{g}_{k, L} \in \operatorname{Cor}(\stackrel{\circ}{L}, D, l)$. Since $D$ is bounded, there exists a subsequence of $\hat{g}_{k, L}$ converging to $\hat{g} \in \operatorname{Cor}(\stackrel{\circ}{L}, \bar{D}, l)$. By exhausting $\Sigma$ with compacts and passing to the diagonal subsequence, we obtain a limit $\hat{g} \in \operatorname{Cor}(\Sigma, \bar{D}, l)$.

To prove that $\hat{f} \in \operatorname{Cor}(D, \Sigma, m)$ and $\hat{g} \in \operatorname{Cor}(\Sigma, D, l)$, we need the following statement, which follows from the Schwarz lemma for proper holomorphic correspondences (see [12]).

Lemma 1. Let $D$ and $G$ be bounded domains in $\mathbb{C}^{n}$ and $(a, b) \in D \times G$. Then for any neighborhood $U_{2} \ni b$ in $G$ there exists a neighborhood $U_{1} \ni$ $a$ in $D$ such that if $h \in \operatorname{Cor}(D, G, m)$ with $b \in h(a)$ then $h_{U_{1}}^{(a, b)}\left(U_{1}\right) \subset U_{2}$. 
Recall that the branches $\left\{\hat{f}^{1}, \ldots, \hat{f}^{m}\right\}$ of $\hat{f}$ are locally defined and holomorphic on $D \backslash \pi_{1}(V)$. Then the Jacobian of $\hat{f}^{i}$ induce in a natural manner a holomorphic function on the graph $\hat{f} \backslash V$ as follows: if $z \in \operatorname{graph} \hat{f} \backslash V$; there exists only one $i \in\{1, \ldots, m\}$ such that $z \in$ graph $\hat{f}^{i} \backslash V$. We define $\operatorname{Jac} \hat{f}(z)=\operatorname{Jac} \hat{f}^{i}\left(\pi_{1} z\right)$.

Claim 1. $\hat{f} \in \operatorname{Cor}(D, \Sigma, m)$.

Proof: Since for any compact $K \ni p$, the correspondence $\hat{f}_{k, K}^{(p, s)}$ is uniformly bounded, so in view of Lemma 1 there exist neighborhoods $U_{2} \ni s$ and $U_{1} \ni p$ such that for all $k, \hat{f}_{k, U_{1}}^{(p, s)}\left(U_{1}\right) \subset U_{2}$. Then we have $z \in$ $\hat{g}_{k, U_{2}}^{(s, p)} \circ \hat{f}_{k, U_{1}}^{(p, s)}(z)$ for all $z \in U_{1}$. Passing to a convergent subsequence, we get $z \in \hat{g}_{U_{2}} \circ \hat{f}_{U_{1}}(z)$ for all $z \in U$, which implies that Jac $\hat{f}_{U_{1}} \not \equiv 0$.

Now assume that there exist points $a \in D$ and $b \in \partial \Sigma$ such that $b \in f(a)$. Let $A_{1}$ be an irreducible component of graph $\hat{f}$ containing $(a, b)$. According to [7], there exist small neighborhoods $U^{\prime} \ni a$, $U^{\prime \prime} \ni b$, so the projection $\pi: A_{1} \cap\left(U^{\prime} \times U^{\prime \prime}\right) \rightarrow U^{\prime}$ is proper. Let $h_{1}, \ldots, h_{k}$ be the branches of $\pi^{-1}$ which are locally defined and holomorphic on $U^{\prime} \backslash \sigma$, with $\sigma$ an analytic set of dimension at most $n-1$. Since $b$ is a strong pseudoconvexity point, there exists a local plurisubharmonic peak function $\psi$ defined in a neighborhood of $b$. Without loss of generality, we may assume that $\psi \in P S H\left(U^{\prime \prime} \cap \Sigma\right) \cap C\left(U^{\prime \prime} \cap \bar{\Sigma}\right)$. Consider $\rho(z)=\max \left(\psi \circ h_{1}(z), \ldots, \psi \circ h_{k}(z)\right)$. It is clear that $\rho$ is plurisubharmonic in $U^{\prime} \backslash \sigma$, since it is bounded $(\psi \leq 1)$, so it extends as a plurisubharmonic function on $U^{\prime}$. But $\rho(a)=1$, then by the maximum principle, $\rho(z) \equiv 1$ on $U^{\prime}$. This implies that one of the branches $h_{i} \equiv b$ on $U^{\prime}$. Hence $U^{\prime} \times\{b\} \subset A_{1}$. It follows by irreducibility that $D \times\{b\}=A_{1}$. Since $A_{1}$ is an arbitrary component containing $(a, b)$, it follows that the only one component of graph $\hat{f}$ containing $(a, b)$ is $D \times\{b\}$. A similar argument shows that for all $z \in D$, the only component of graph $\hat{f}$ containing $(z, b)$ is $D \times\{b\}$. Therefore, if $A_{j}$ is some component of graph $\hat{f}$ distinct from $D \times\{b\}$, then $A_{j} \cap(D \times\{b\})=\emptyset$. This shows that for all $z \in D, \hat{f}(z)=\left\{\hat{f}_{1}(z), \ldots, \hat{f}_{m-1}(z), b\right\}$. But the branches of $\hat{f}$ are locally open on $U_{1} \backslash\left(\pi_{1}\{V \cup W\} \cap U_{1}\right)$, since Jac $\hat{f}_{U_{1}} \not \equiv 0$. This contradiction completes the proof of Claim 1 .

Claim 2. $\hat{g} \in \operatorname{Cor}(\Sigma, D, l)$.

Proof: Since the domain $D$ is bounded, we can repeat the same proof of W. Klingenberg and S. Pinchuk [12] (see also [17]) to show that 
$\hat{g} \in \operatorname{Cor}(\Sigma, D, l)$. For the convenience of the reader we include this proof.

The same argument used for the correspondence $\hat{f}$ shows that $\operatorname{Jac} \hat{g}_{U(s)} \not \equiv 0$ for a certain neighborhood $U(s)$ of $s$. It follows that $\operatorname{Jac} \hat{g} \not \equiv 0$. Now assume that the claim is false, i.e. there exists $(x, y) \in$ $\Sigma \times \partial D$ with $y \in \hat{g}(x)$. Since the branches of $\hat{g}$ are locally open maps on $\Sigma \backslash \pi_{1}\left\{V^{\prime} \cup W^{\prime}\right\} \quad\left(V^{\prime}\right.$ is the branch locus of $\hat{g}$ and $W^{\prime} \subset$ graph $\hat{g} \backslash V^{\prime}$ denotes the variety $\{\operatorname{Jac} \hat{g}=0\})$, we must have $x \in \pi_{1}\left\{V^{\prime} \cup W^{\prime}\right\}$. Let $\tilde{\Delta}$ a holomorphic disc in $\Sigma$ such that $\tilde{\Delta} \cap \pi_{1}\left\{V^{\prime} \cup W^{\prime}\right\}=\{x\}$. As $\hat{g}(\tilde{\Delta}) \subset D \cup\{y\}$ is a disc, by the theorem of Cartan-Thullen (see [28]), the correspondences $\hat{f}_{k}$ and $\hat{f}$ extend analytically to a fixed neighborhood of $y$, say $U(y)$. The domain $\Sigma$ is biholomorphic to the unit ball which is a bounded domain, then there exists a subsequence of $\hat{f}_{k}$ which converges to $\hat{f}$ on the compact subsets of $D \cup U(y)$. It follows from the assumption that there exists $y_{k} \in \hat{g}_{k}(x)$ with $y_{k} \rightarrow y$. But since $\hat{g}_{k}$ is the inverse of $\hat{f}_{k}$, this implies that $x \in \hat{f}_{k}\left(y_{k}\right)$, and we may pass to the limit, which gives $x \in \hat{f}(y)$. Since $\hat{f}_{k}$ is proper, we have $\hat{f}_{k}(y) \subset \partial \hat{G}^{k}$ and then by passing to a convergent subsequence and to the limit, we obtain $\hat{f}(y) \subset \partial \Sigma$. This contradicts $x \in \Sigma$.

Now, we shall prove that $\hat{f}$ is proper. First we prove that the correspondence $\hat{g}$ is the inverse of $\hat{f}$. Let $(a, b) \in$ graph $\hat{f}$. Then there exists a sequence $\left\{b_{k}\right\}_{k}, b_{k} \in \hat{f}_{k}(a)$ such that $b_{k} \rightarrow b$. Since the correspondence $\hat{g}_{k}$ is the inverse of the correspondence $\hat{f}_{k}$, we have $a \in \hat{g}_{k}\left(b_{k}\right)$. Passing to a convergent subsequence and to the limit we get $a \in \hat{g}(b)$. The same argument proves that if $(b, a) \in \operatorname{graph} \hat{g}$ then $(a, b) \in \operatorname{graph} \hat{f}$.

Let $\left\{z_{j}\right\}_{j}$ be a sequence in $D$ that converges to a point $z \in \partial D$. By contradiction assume that $\hat{f}$ is not proper. Then (after passing to a subsequence), there exists a sequence $\left\{w_{j}\right\}_{j}$ in $\Sigma$ with $w_{j} \in \hat{f}\left(z_{j}\right)$ that converges to $w \in \Sigma$. Since $\hat{g}$ is the inverse of $\hat{f}$, we have $z_{j} \in \hat{g}\left(w_{j}\right)$. This implies $z \in \hat{g}(w) \subset D$. This contradiction proves that $\hat{f}$ is proper and completes the proof of Theorem 1.

Proof of Theorem 2: According to Theorem 1 there exists a proper holomorphic correspondence $F$ from $D$ onto $\mathbb{B}$. In view of $[\mathbf{3}]$ and $[\mathbf{1 8}]$ each branch of the correspondence $f_{k} \circ F^{-1}: \mathbb{B} \rightarrow G$ defines a biholomorphic mapping. Then $G$ is biholomorphic to the unit ball.

Proof of Corollary 1: Without loss of generality we may assume that the domain $G$ is strongly pseudoconvex (otherwise, we consider the correspondence inverse $f_{k}^{-1}$ ). Fix $p$ a point in $D$ and set $q_{k} \in f_{k}(p)$. Since $G$ 
is bounded we can assume (after passing to a subsequence) that $\left\{q_{k}\right\}_{k}$ converges to a point $q \in \bar{G}$. If $q \in \partial G$, then Theorem 2 implies that $G$ is biholomorphic to the unit ball. Consequently, the point $q \in G$. Thus the correspondence $\left\{f_{k}\right\}_{k}$ is not compactly divergent. According to [12], we can extract a subsequence of $\left\{f_{k}\right\}_{k}$ converging to a proper holomorphic correspondence in $\operatorname{Cor}(D, G, m)$.

Proof of Corollary 2: (1) In view of [13] $M$ is a Kobayashi hyperbolic. Since $M$ is homogeneous, according to [15] $M$ is biholomorphically equivalent to a bounded domain $D$ of $\mathbb{C}^{n}$. The nonexistence of proper holomorphic mapping from $D$ onto $G$ was proved in [17]. Now we shall prove that the same conclusion holds for proper holomorphic mappings from $G$ onto $D$. Fix $p \in D$ a point and $q \in \partial G$ a strongly pseudoconvex boundary point. Let $\left\{q_{k}\right\}_{k}$ be a sequence of points of $G$ that converges to $q$ and $f$ a proper holomorphic mapping from $G$ onto $D$ with multiplicity $m$. Since $D$ is homogeneous, there exists a sequence of automorphisms $\left\{\varphi_{k}\right\}_{k} \subset \operatorname{Aut}(D)$ such that $\varphi_{k}(p)=f\left(q_{k}\right)$. Thus $q_{k} \in f^{-1} \circ \varphi_{k}(p)$. Theorem 1 implies that there exists a proper holomorphic correspondence in $\operatorname{Cor}(D, \mathbb{B}, m, 1)$. Then there exists a proper holomorphic mapping $P$ from $\mathbb{B}$ onto $D$. In view of $[\mathbf{2 3}]$ we may assume that $P$ is a polynomial mapping. Then $P$ is a biholomorphism in a neighborhood of any points $z \in \partial \mathbb{B}$ with $\operatorname{Jac}(P)(z) \neq 0$. From this we conclude that $D$ contains strong pseudoconvexity boundary points. By the Wong-Rosay theorem $D$ is biholomorphic to the unit ball.

(2) Let $f_{k}$ be a correspondence in $\operatorname{Cor}(M, G, m, l)$. The same argument as above implies the existence of a proper holomorphic correspondence from $M$ onto the unit ball. From this we conclude $G$ is biholomorphic to the unit ball. If $f_{k}$ is in $\operatorname{Cor}(G, M, m, l)$, we consider $f_{k}^{-1}$ and the proof is similar.

\section{References}

[1] E. Bedford And S. Bell, Boundary continuity of proper holomorphic correspondences, in: "Séminaire d'analyse P. LelongP. Dolbeault-H. Skoda, années 1983/1984", Lecture Notes in Math. 1198, Springer, Berlin, 1986, pp. 47-64.

[2] E. BEDFord AND S. BelL, Holomorphic correspondences of bounded domains in $\mathbb{C}^{n}$, in: "Complex analysis" (Toulouse, 1983), Lecture Notes in Math. 1094, Springer, Berlin, 1984, pp. 1-14.

[3] E. Bedford and S. Bell, Boundary behavior of proper holomorphic correspondences, Math. Ann. 272(4) (1985), 505-518. 
[4] E. Bedford And S. Pinchuk, Domains in $\mathbb{C}^{n+1}$ with noncompact automorphism group, J. Geom. Anal. 1(3) (1991), 165-191.

[5] S. BELL, A generalization of Cartan's theorem to proper holomorphic mappings, J. Math. Pures Appl. (9) 67(1) (1988), 85-92.

[6] F. Berteloot and A. Sukhov, On the continuous extension of holomorphic correspondences, Ann. Scuola Norm. Sup. Pisa Cl. Sci. (4) 24(4) (1997), 747-766.

[7] E. M. ChIRKA, "Complex analytic sets", Mathematics and its Applications (Soviet Series) 46, Kluwer Academic Publishers Group, Dordrecht, 1989.

[8] B. Coupet, S. Pinchuk And A. Sukhov, On boundary rigidity and regularity of holomorphic mappings, Internat. J. Math. 7(5) (1996), 617-643.

[9] B. Coupet And A. Sukhov, On CR mappings between pseudoconvex hypersurfaces of finite type in $\mathbb{C}^{2}$, Duke Math. J. 88(2) (1997), 281-304.

[10] J. P. D'Angelo, Real hypersurfaces, orders of contact, and applications, Ann. of Math. (2) 115(3) (1982), 615-637.

[11] S. Frankel, Complex geometry of convex domains that cover varieties, Acta Math. 163(1-2) (1989), 109-149.

[12] W. Klingenberg And S. Pinchuk, Normal families of proper holomorphic correspondences, Math. Z. 207(1) (1991), 91-96.

[13] S. LANG, "Introduction to complex hyperbolic spaces", SpringerVerlag, New York, 1987.

[14] E. B. Lin And B. Wong, Curvature and proper holomorphic mappings between bounded domains in $\mathbb{C}^{n}$, Rocky Mountain J. Math. 20(1) (1990), 179-197.

[15] K. NAKAJima, Homogeneous hyperbolic manifolds and homogeneous Siegel domains, J. Math. Kyoto Univ. 25(2) (1985), 269-291.

[16] K. Oelueklaus And E. H. Youssfi, Proper holomorphic mappings and related automorphism groups, J. Geom. Anal. 7(4) (1997), 623-636.

[17] N. OURIMI, A local version of Wong-Rosay's theorem for proper holomorphic mappings, Proc. Amer. Math. Soc. 128(3) (2000), 831-836.

[18] S. Pinchuk, Holomorphic inequivalence of certain classes of domains in $\mathbb{C}^{n}$, Mat. Sb. (N.S.) 111(153), no. 1 (1980), 67-94, 159 (in Russian).

[19] S. Pinchuk, The scaling method and holomorphic mappings, in: "Several complex variables and complex geometry, Part 1" (Santa 
Cruz, CA, 1989), Proc. Sympos. Pure Math. 52, Part 1, Amer. Math. Soc., Providence, RI, 1991, pp. 151-161.

[20] S. Pinchuk, Boundary behavior of analytic sets and algebroid mappings, Dokl. Akad. Nauk SSSR 268(2) (1983), 296-298 (in Russian).

[21] J.-P. Rosay, Sur une caractérisation de la boule parmi les domaines de $\mathbb{C}^{n}$ par son groupe d'automorphismes, Ann. Inst. Fourier (Grenoble) 29(4) (1979), 91-97.

[22] H. Rossi, Holomorphically convex sets in several complex variables, Ann. of Math. (2) 74 (1961), 470-493.

[23] W. Rudin, Proper holomorphic maps and finite reflection groups, Indiana Univ. Math. J. 31(5) (1982), 701-720.

[24] N. Sibony, Some aspects of weakly pseudoconvex domains, in: "Several complex variables and complex geometry, Part 1" (Santa Cruz, CA, 1989), Proc. Sympos. Pure Math. 52, Part 1, Amer. Math. Soc., Providence, RI, 1991, pp. 199-231.

[25] K. Stein, Maximale holomorphe und meromorphe Abbildungen. II, Amer. J. Math. 86 (1964), 823-868.

[26] K. Stein, Topics on holomorphic correspondences, Rocky Mountain J. Math. 2(3) (1972), 443-463.

[27] K. Verma, Boundary regularity of correspondences in $\mathbb{C}^{2}$, Math. Z. 231(2) (1999), 253-299.

[28] V. S. Vladimirov, "Methods of the theory of functions of many complex variables", The M.I.T. Press, Cambridge, Mass.-London, 1966.

[29] B. Wong, Characterization of the unit ball in $\mathbb{C}^{n}$ by its automorphism group, Invent. Math. 41(3) (1977), 253-257.

Faculté des Sciences de Bizerte

7021 Jarzouna (Bizerte)

Tunisie

E-mail address: ourimin@yahoo.com

Primera versió rebuda el 26 de juliol de 2001, darrera versió rebuda el 4 de setembre de 2002. 